\title{
Radical surgery for ovarian carcinosarcoma - a case report and literature review
}

\author{
N. Bacalbașa ${ }^{1}$, Irina Bălescu², V. Brașoveanu ${ }^{3}$
}

Corresponding author:

Nicolae Bacalbasa,

Dimitrie Racovita Street, no. 2,

Bucharest, Romania

E-mail: nicolae_bacalbasa@yahoo.ro

\author{
"'Carol Davila " University of Medicine and Pharmacy, Bucharest, Romania \\ 2"Ponderas Hospital" Bucharest, Romania \\ 3Department of General Surgery and Liver Transplantation "Dan Setlacec" \\ Fundeni Clinical Institute, Bucharest, Romania
}

\begin{abstract}
Ovarian carcinosarcomas are rare gynecologic malignancies associated with an overall poor survival. The best way to obtain a good control of the disease seems to be complete cytoreduction; association of adjuvant platinum based chemotherapy was extrapolated from therapeutic guidelines for advanced epithelial ovarian cancer and seems to be effective in carcinosarcomas too. However the most important prognostic factor remains complete cytoreduction at the moment of diagnosis. We present the case of a 55 years old patient diagnosed with stage IIIC ovarian cancer in whom an R0 resection was performed. The histopathological findings revealed the presence of ovarian carcinosarcoma. At an one year follow up the patient is free of any recurrent disease.
\end{abstract}

Key words: ovarian carcinosarcoma, advanced stage, $\mathrm{R} 0$ resection

\section{INTRODUCTION}

Ovarian carcinosarcomas are rare but very aggressive gynecologic malignancies accounting for 1-4\% of all ovarian malignancies with a median survival of less than 18 months (1-3). They are also known as malignant mixed mullerian tumors and consist in the association of an epithelial malignant and a sarcomatous component. (4) Due to the rarity of these tumors there are limited therapeutic protocols, most of them being extrapolated from larger experience with advanced epithelial ovarian cancer. (4) The most important prognostic factors are related to the stage of the disease at the moment of diagnosis and to the completeness of the surgical resection at the time of operation. Patients with initial advanced stage of the disease and incomplete cytoreduction report the poorest outcome. (5) At the moment of presentation up to $75 \%$ of cases will have widespread metastases (FIGO stages III-IV) which are usually associated with a poor 5 year survival of only $7-20 \%(1-3 ; 6 ; 7)$. We present the case of a 55 year old female diagnosed with stage IIIC ovarian cancer in whom complete debulking surgery was performed and in whom the histopathological findings revealed an ovarian carcinosarcoma. 


\section{CASE REPORT}

The 55 years old patient presented for weight loss and pelvic pain and was diagnosed at pre-operative imaging studies with a large ovarian tumor associated with widespread tumoral peritoneal nodules. The patient was submitted to surgery and a radical total hysterectomy en bloc with bilateral adnexectomy, rectosigmoidectomy, pelvic and para-aortic lymph node dissection, omentectomy and total peritonectomy were performed. (figures 1-5) The histopathological findings revealed an ovarian carcinosarcoma. (figure 6) The postoperative evolution was marked by the apparition of a pleural effusion and pleurostomy was placed. Cytology from the pleural effusion showed no tumoral cells. The pleal drainage tube was removed in the 6th postoperative day and the patient was discharged 2 days later. The patient was subsequently submitted to oncologic treatment with taxanes and platinum salts. One year after finishing the oncologic treatment she is free of any recurrent disease.

\section{DISCUSSIONS}

Ovarian carcinosarcomas are very aggressive malignancies associated with a grim prognosis. They are also known as mixed mullerian tumors comprising both an epithelial and a sarcomatous component. Nowadays there are three theories which explain the pathogenesis and evolution of these tumors. The first one sustains the idea that there are two different malignant cells - an epithelial one and a sarcomatous one which evolve separately then they collide (collision theory); the second one sustains the idea of o single malignant stem cell which gives birth to both an epithelial and a sarcomatous malignant cell (the combination theory); the last theory, also called the conversion

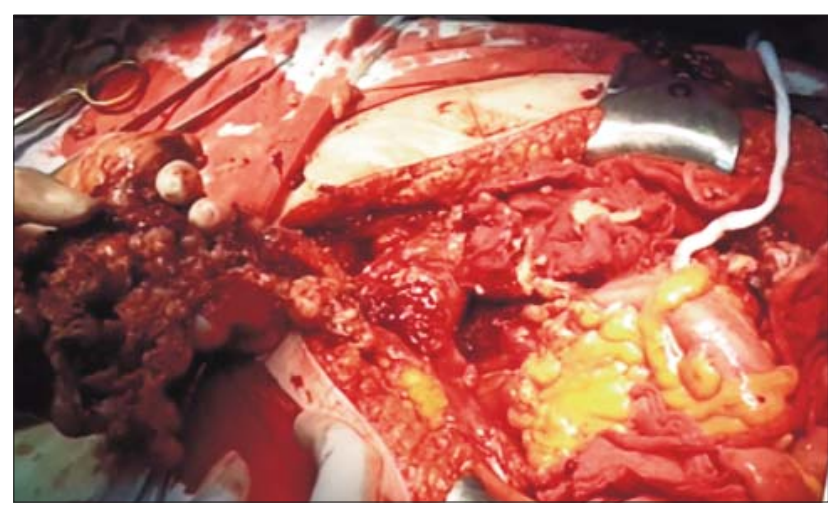

Figure 1 - large ovarian tumor associated with peritoneal nodules

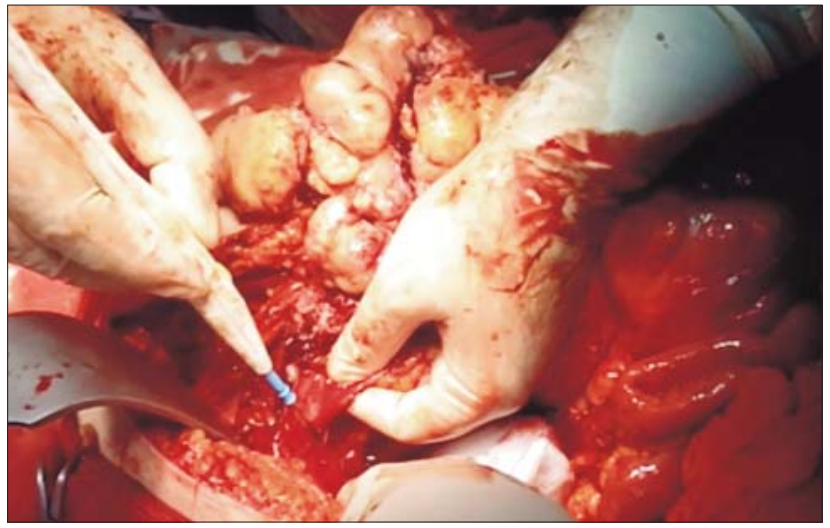

Figure 2 - parietal peritonectomy

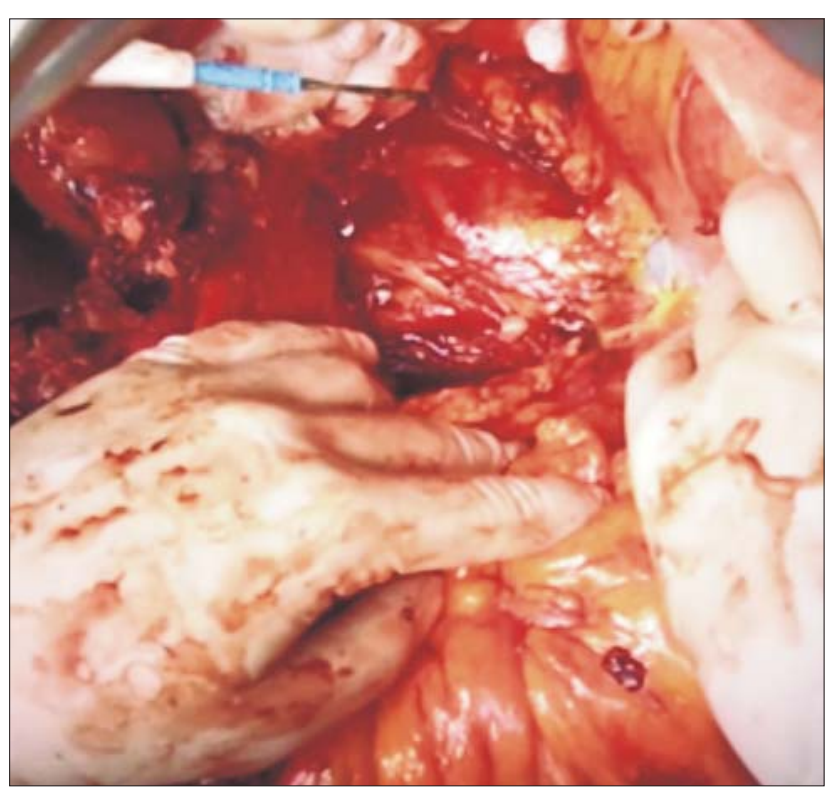

Figure 3 - removing the paracaval lymph nodes

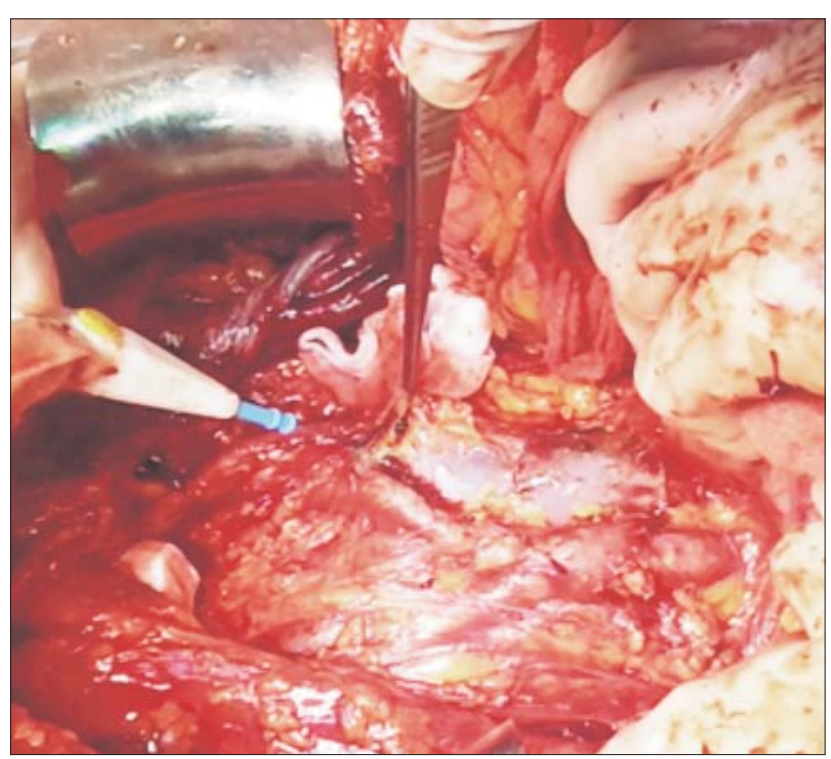

Figure 4 - inter aortico-caval lymph node dissection 


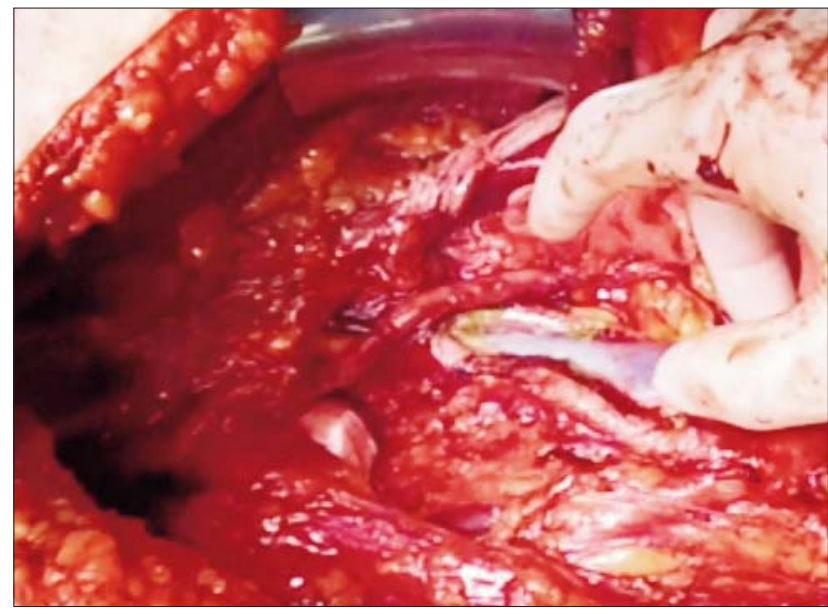

Figure 5 - the final aspect after total radical hysterectomy with bilateral adnexectomy, rectosigmoidectomy, parietal peritonectomy, pelvic and para-aortic lymph node dissection

theory affirms that there is a single cell which differentiates first into one cell type and then it transforms in the other cell type. $(5,8)$ However, ovarian carcinosarcomas have some common features with epithelial ovarian cancer but they are also distinctly different from the epithelial tumors. (9)

Due to the lack of studies involving large series of patients diagnosed with ovarian carcinosarcomas it is hard to establish which is the most appropriate therapeutical protocol for these patients. Some authors extrapolated the results obtained in treating advanced epithelial ovarian cancer to those diagnosed with ovarian carcinosarcomas. (4) However it seems that cases diagnosed with ovarian carcinosarcomas benefit most from an aggressive surgical approach combined with adjuvant chemotherapy.

The largest series of ovarian carcinosarcomas was studied by D. Doo and included 51 patients. In this study the authors demonstrated that both disease free survival and overall survival were significantly improved in patients who underwent complete cytoreduction (Median disease free survival varied significantly among groups: 29 months in the group who underwent Ro resection versus 21 months in the group with residual disease $<1 \mathrm{~cm}$ versus 2 months for the cases who had residual disease $>1 \mathrm{~cm}(p=0.036)$; the same difference was seen when comparing the median overall survivals too: 57 vs. 32 vs. 11 months ( $p=0.015)$. When patients with stage 3 disease were compared separately,median OS still varied significantly among groups: 57 versus 31 versus 3 months $(p=0.009)$. (4)

These results come to contradict the first reports conducted on cases with ovarian carcinosarcomas who failed to demonstrate the direct correlation between

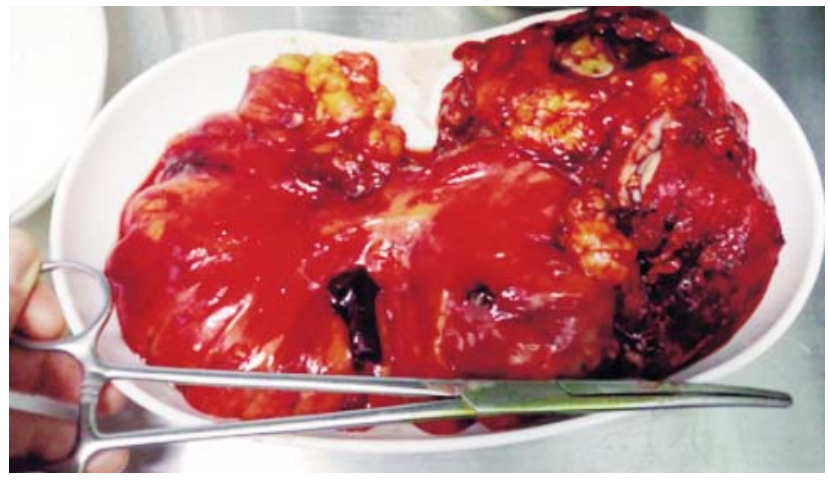

Figure 6 - the specimen; the histopathological exam revealed the presence of an ovarian carcinosarcoma.

complete cytoreduction and overall survival. In Barakat's study the size of the residual tumor did not significantly impacted on overall survival. (10)

In order to demonstrate both the efectiveness of complete cytoreduction and the different postoperative outcomes for ovarian carcinosarcomas and epithelial ovarian cancer, Rauh-Hain et al realized a case control study conducted on 50 patients. They compared the evolution, the response to chemotherapy and survival of patients with ovarian carcinosarcoma to those with epithelial ovarian cancer with similar features. A group of 50 patients diagnosed with ovarian carcinosarcoma was compared to a similar group formed by 100 patients with the same features diagnosed with epithelial ovarian cancer. Each patient in the first group had 2 corresponding matches in the other group with similar age, stage of the disease and year of diagnosis. All patients underwent adjuvant chemotherapy with taxanes and platinum salts; the rates of response were $62 \%$ for cases with carcinosarcomas and 83\% for epithelial adenocarcinomas $(p=0,003)$ while median progression-free survival was 11 months versus 16 months ( $p=0.02)$ and median overall survival was 24 months versus 41 months $(p=0.002)$ for cases and controls, respectively. The 50 cases with ovarian carcinosarcomas were analysed separately too and significant statistical differences were obtained between groups who were submitted to complete cytoreduction when compared to those with residual disease $<1 \mathrm{~cm}$ or $>1 \mathrm{~cm}$ respectively. DFS for women with $\mathrm{RO}$ resection was 19 months compared to a DFS of 10 months in patients with optimal but macroscopic residual tumor, and the median DFS was 5 months in cases with suboptimal cytoreduction $(p=0.01)$. The median OS for women with only RO resection was 47 months compared to an OS of 18 months in the patients with optimal but macroscopic residual tumor, and 8 months in those patients with suboptimal cytoreduction $(p=0.02)$. (11) 
Similar rates of response to taxanes-platinum salts regimens (which are lower than those obtained for epithelial ovarian cancer) were reported by other studies too. In a cohort of 30 patients Leiser et al reported a rate of response to this regimen of chemotherapy of $63 \%$. (12) while Duska et al reported a total response rate of $72 \%$ (13). The mechanisms responsible for a more aggressive course for ovarian carcinosarcomas are not known yet but it seems that the poorer response to adjuvant chemotherapy plays an important role. (10)

The similarities between ovarian carcinosarcomas and uterine sarcomas raised the question of the utility of the association between ifosfamide and platinum salts. One of the largest studies which sustained this chemotherapeutic protocol was conducted by Crotzer et al and included 8 patients. They reported a median overall survival of 21 months with acceptable rates of ifosfamide related toxicity. (14)

\section{CONCLUSIONS}

Although ovarian carcinosarcomas represent rather a discouraging pathology, latest studies have demonstrated that an aggressive surgical approach is perfectly justified in order to obtain a satisfactory control of the disease. It seems that complete cytoreduction was responsible for a good long term outcome in our case too. However there are still important aspects to be discussed in this aggressive gynaecological malignancy. Unfortunately, the most important fact that impedes studies in this malignancy is the small number of patients included in the present studies and the lack of prospective analyses.

\section{REFERENCES}

1. Barnholtz-Sloan,J.S., Morris,R., Malone,J.M., Jr., and Munkarah,A.R. 2004. Survival of women diagnosed with malignant, mixed mullerian tumors of the ovary (OMMMT). Gynecol. Oncol. 93:506-512.

2. Mano,M.S., Rosa,D.D., Azambuja,E., Ismael,G., Braga,S., D'Hondt,V., Piccart,M., and Awada,A. 2007. Current management of ovarian carcinosarcoma. Int. J. Gynecol. Cancer 17:316-324.

3. Harris,M.A., Delap,L.M., Sengupta,P.S., Wilkinson,P.M., Welch,R.S., Swindell,R., Shanks,J.H., Wilson,G., Slade,R.J., Reynolds,K. et al 2003. Carcinosarcoma of the ovary. Br. J. Cancer 88:654-657.

4. Doo,D.W., Erickson,B.K., Arend,R.C., Conner,M.G., Huh,W.K., and Leath,C.A., III 2014. Radical surgical cytoreduction in the treatment of ovarian carcinosarcoma. Gynecol. Oncol. 133:234-237.

5. Cantrell,L.A., and Van,L.L. 2009. Carcinosarcoma of the ovary a review. Obstet. Gynecol. Surv. 64:673-680.

6. Rutledge,T.L., Gold,M.A., McMeekin,D.S., Huh,W.K., Powell,M.A., Lewin,S.N., Mutch,D.G., Johnson,G.A., Walker,J.L., and Mannel,R.S. 2006. Carcinosarcoma of the ovary-a case series. Gynecol. Oncol. 100:128-132.

7. Brown,E., Stewart,M., Rye,T., Al-Nafussi,A., Williams,A.R., Bradburn,M., Smyth,J., and Gabra,H. 2004. Carcinosarcoma of the ovary: 19 years of prospective data from a single center. Cancer 100:2148-2153.

8. Inthasorn,P., Beale,P., Dalrymple,C., and Carter,J. 2003. Malignant mixed mullerian tumour of the ovary: prognostic factor and response of adjuvant platinum-based chemotherapy. Aust. N. Z. J. Obstet. Gynaecol. 43:61-64.

9. del Carmen,M.G., Birrer,M., and Schorge,J.0. 2012. Carcinosarcoma of the ovary: a review of the literature. Gynecol. Oncol. 125:271-277.

10. Barakat,R.R., Rubin,S.C., Wong,G., Saigo,P.E., Markman,M., and Hoskins, W.J. 1992. Mixed mesodermal tumor of the ovary: analysis of prognostic factors in 31 cases. Obstet. Gynecol. 80:660-664.

11. Rauh-Hain,J.A., Growdon,W.B., Rodriguez,N., Goodman,A.K., Boruta, D.M., Schorge,J.O., Horowitz,N.S., and del Carmen,M.G. 2011. Carcinosarcoma of the ovary: a case-control study. Gynecol. Oncol. 121:477-481.

12. Leiser,A.L., Chi,D.S., Ishill,N.M., and Tew,W.P. 2007. Carcinosarcoma of the ovary treated with platinum and taxane: the memorial Sloan-Kettering Cancer Center experience. Gynecol. Oncol. 105:657-661.

13. Duska,L.R., Garrett,A., Eltabbakh,G.H., Oliva,E., Penson,R., and Fuller,A.F. 2002. Paclitaxel and platinum chemotherapy for malignant mixed mullerian tumors of the ovary. Gynecol. Oncol. 85:459-463.

14. Crotzer,D.R., Wolf,J.K., Gano,J.B., Gershenson,D.M., and Levenback,C. 2007. A pilot study of cisplatin, ifosfamide and mesna in the treatment of malignant mixed mesodermal tumors of the ovary. Gynecol. Oncol. 105:399-403. 